\title{
Transverse-momentum resummation and the structure of hard factors at the NNLO
}

\author{
Leandro Cieri* \\ Universita di Roma "La Sapienza" \\ E-mail: Cieridromal.infn.it
}

In this proceeding we consider QCD radiative corrections to the production of colourless highmass systems in hadron collisions. At small transverse momentum the logarithmically-enhanced contributions can be organized to all perturbative orders by a universal resummation formula that depends on a single process-dependent hard factor. We show that the hard factor is directly related to the all-order virtual amplitude of the corresponding partonic process by a universal (process independent) formula, which we explicitly evaluate up to two-loop level. Once the next-to-next-toleading order (NNLO) scattering amplitude is available, the corresponding hard factor is directly determined. It can be used in fully-exclusive perturbative calculations ( $v i a q_{T}$ subtraction formalism) up to NNLO, in resummed calculations at full next-to-next-to-leading logarithmic (NNLL) accuracy, and also, it's a necessary ingredient to the next subsequent logarithmic order $\left(\mathrm{N}^{3} \mathrm{LL}\right)$.

Loops and Legs in Quantum Field Theory - LL 2014,

27 April - 2 May 2014

Weimar, Germany

\footnotetext{
* Speaker.
} 


\section{Introduction}

We consider the inclusive hard-scattering reaction

$$
h_{1}\left(p_{1}\right)+h_{2}\left(p_{2}\right) \rightarrow F\left(\left\{q_{i}\right\}\right)+X,
$$

where the collision of the two hadrons $h_{1}$ and $h_{2}$ with momenta $p_{1}$ and $p_{2}$ produces the observed final state $F$, accompanied by an arbitrary and undetected final state $X$. The triggered final state $F$ is a generic system of one or more colourless particles, such as lepton pairs (produced by DrellYan (DY) mechanism), photon pairs, vector bosons, Higgs boson(s), and so forth. The momenta of these final state particles are denoted by $q_{1}, q_{2} \ldots q_{n}$. The system $F$ has total invariant mass $M^{2}=\left(q_{1}+q_{2}+\ldots q_{n}\right)^{2}$, transverse momentum $\mathbf{q}_{\mathbf{T}}$ and rapidity $y$. We employ $\sqrt{s}$ to denote the centre-of-mass energy of the colliding hadrons, which are treated in the massless approximation $\left(s=\left(p_{1}+p_{2}\right)^{2}=2 p_{1} \cdot p_{2}\right)$.

It is possibile to calculate the transverse-momentum $\left(q_{T}\right)$ cross section for the process in Eq. (미) by using perturbative QCD. In the small- $q_{T}$ region (roughly, in the region where $q_{T} \ll M$ ) the convergence of the fixed-order perturbative expansion in powers of the QCD coupling $\alpha_{\mathrm{S}}$ is spoiled by the presence of large logarithmic terms of the type $\ln ^{n}\left(M^{2} / q_{T}^{2}\right)$. We can recover the predictivity of perturbative QCD performing the summation of these logarithmically-enhanced contributions to all order in $\alpha_{\mathrm{S}}$ [四, 口, []].

If the final state $F$ is colourless, the large logarithmic contributions to the $q_{T}$ cross section can be systematically resummed to all perturbative orders, and the structure of the resummed calculation can be arranged in a process-independent form [ [1, [], [1, 6]. Starting from the resummation formula for the DY process [వ] , two additional steps were needed to arrive at the process-independent version of the formalism: the understanding of the all-order process-independent structure of the Sudakov form factor (through the factorization of a single process-dependent hard factor) [\$]], and the complete generalization to processes that are initiated by the gluon fusion mechanism [ [6].

The all-order process-independent form of the resummed calculation has a factorized structure, whose resummation factors are (see Sect. 『) the (quark and gluon) Sudakov form factor, processindependent collinear factors and a process-dependent hard or, more precisely (see Sect. B]), hardvirtual factor. These factors (which are a set of perturbative functions whose perturbative resummation coefficients are computable order-by-order in $\alpha_{\mathrm{S}}$ ) control the resummation of the logarithmic contributions. The perturbative coefficients of the Sudakov form factor are known, since some time [3], प, 田, [8], up to the second order in $\alpha_{\mathrm{S}}$, and the third-order coefficient $A^{(3)}$ (which is necessary to explicitly perform resummation up to the next-to-next-to-leading logarithmic (NNLL) accuracy) is also known [9]. The next-to-next-to-leading order (NNLO) QCD calculation of the $q_{T}$ cross section (in the small- $q_{T}$ region) has been done in analytic form for two benchmark processes, namely, SM

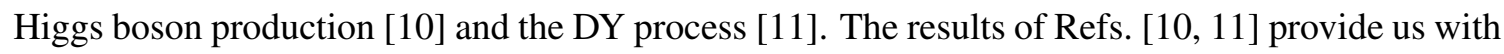
the complete knowledge of the process-independent collinear resummation coefficients up to the second order in $\alpha_{\mathrm{S}}$, and with the explicit expression of the hard coefficients for these two specific processes. As shown in Ref. [12], the hard factor (which is process dependent) has an universal (process-independent) structure. The universality structure of the factorization formula has a soft (and collinear) origin, and it is closely (though indirectly) related to the universal structure of the infrared divergences [[13] of the scattering amplitude. This process-independent structure of the 
hard-virtual term, which generalizes the next-to-leading order (NLO) results of Ref. [ 8$]$, is valid to all perturbative orders [12].

The NNLO universal formula for the hard-virtual term completes the $q_{T}$ resummation formalism in explicit form up to full NNLL+NNLO accuracy. This permits direct applications to NNLL+NNLO resummed calculations for any processes of the class in Eq. (미) (provided the corresponding NNLO amplitude is known), as already done for the cases of SM Higgs boson [प4] and DY [15], [16] production. The NNLO information of the $q_{T}$ resummation formalism is also relevant in the context of fixed order calculations. Indeed, it enables to carry out fully-exclusive NNLO calculations by applying the $q_{T}$ subtraction formalism of Ref. [피] (the subtraction counterterms of the formalism follow [17] from the fixed-order expansion of the $q_{T}$ resummation formula, as in Sect. 2.4 of Ref. [[4]]). The $q_{T}$ subtraction formalism has been applied to the NNLO computation of Higgs boson [ㅍ, [8] and vector boson production [ㅍ] , associated production of the Higgs boson with a $W$ boson [20], diphoton production [[2]], $Z \gamma$ production [[2] and $Z Z$ production [[23]. The computations of Refs. [ए], [18, [Q], [20] were based on the specific calculation of the NNLO hardvirtual coefficients of the corresponding processes [[0, प]]. The computations of Refs. [2], 22, 22] ] used the NNLO hard-virtual coefficients that are determined by applying the universal form of the hard-virtual term that is derived in [ए2] and illustrated in the present proceeding.

Transverse-momentum resummation can equivalently be reformulated by using $q_{T}$-dependent partonic distributions (see, e.g., Refs. [Q, [24]). The explicit NNLO results for the process-independent

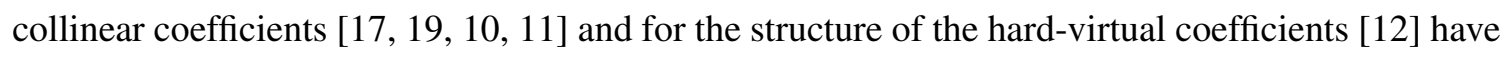
been confirmed by the fully-independent computation of Ref. [[25], which uses the formalism of Ref. [Q].

\section{Small- $q_{T}$ resummation}

We consider the inclusive-production process in Eq. ([I) , and we introduce the corresponding fully differential cross section ${ }^{1}$

$$
\frac{d \sigma_{F}}{d^{2} \mathbf{q}_{\mathbf{T}} d M^{2} d y d \Omega}\left(p_{1}, p_{2} ; \mathbf{q}_{\mathbf{T}}, M, y, \Omega\right),
$$

which depends on the total momentum of the system $F$ (i.e. on the variables $\mathbf{q}_{\mathbf{T}}, M, y$ ). To evaluate the $\mathbf{q}_{\mathbf{T}}$ dependence of the differential cross section in Eq. (D.T) within QCD perturbation theory, we first propose the following decomposition:

$$
d \sigma_{F}=d \sigma_{F}^{(\text {sing })}+d \sigma_{F}^{(\mathrm{reg})} .
$$

The two last terms in the right-hand side already include the convolutions of partonic cross sections and the scale-dependent parton distributions $f_{a / h}\left(x, \mu^{2}\right)\left(a=q_{f}, \bar{q}_{f}, g\right.$ is the parton label) of the colliding hadrons. We use parton densities as defined in the $\overline{\mathrm{MS}}$ factorization scheme, and $\alpha_{\mathrm{S}}\left(q^{2}\right)$ is the QCD running coupling in the $\overline{\mathrm{MS}}$ renormalization scheme. The partonic cross sections that enter the singular component (the first term in the right-hand side of Eq. (‥2)) contain all the contributions that are enhanced (or 'singular') at small $q_{T}$. These contributions are proportional

\footnotetext{
${ }^{1}$ In this section we briefly recall the formalism of transverse-momentum resummation in impact parameter space [四, 四, 田, 回, 目]. We closely follow the notation of Ref. [目] (more details about our notation can be found therein).
} 
to $\delta^{(2)}\left(\mathbf{q}_{\mathbf{T}}\right)$ or to large logarithms of the type $\frac{1}{q_{T}^{2}} \ln ^{m}\left(M^{2} / q_{T}^{2}\right)$. The partonic cross sections of the second term in the right-hand side of Eq. (2.2) are regular (i.e. free of logarithmic terms) orderby-order in perturbation theory as $q_{T} \rightarrow 0$. In the following we focus on the singular component, $d \sigma_{F}^{\text {(sing) }}$, which has an universal all-order structure. The corresponding resummation formula is written as [ய, [1, 目]

$$
\begin{aligned}
\frac{d \sigma_{F}^{(\text {sing })}\left(p_{1}, p_{2} ; \mathbf{q}_{\mathbf{T}}, M, y, \Omega\right)}{d^{2} \mathbf{q}_{\mathbf{T}} d M^{2} d y d \Omega} & =\frac{M^{2}}{s} \sum_{c=q, \bar{q}, g}\left[d \sigma_{c \bar{c}, F}^{(0)}\right] \int \frac{d^{2} \mathbf{b}}{(2 \pi)^{2}} e^{i \mathbf{b} \cdot \mathbf{q}_{\mathbf{T}}} S_{c}(M, b) \\
& \times \sum_{a_{1}, a_{2}} \int_{x_{1}}^{1} \frac{d z_{1}}{z_{1}} \int_{x_{2}}^{1} \frac{d z_{2}}{z_{2}}\left[H^{F} C_{1} C_{2}\right]_{c \bar{c} ; a_{1} a_{2}} f_{a_{1} / h_{1}} f_{a_{2} / h_{2}},
\end{aligned}
$$

where $b_{0}=2 e^{-\gamma_{E}}\left(\gamma_{E}=0.5772 \ldots\right.$ is the Euler number $)$ is a numerical coefficient, and the kinematical variables $x_{1}=\frac{M}{\sqrt{s}} e^{+y}$ and $x_{2}=\frac{M}{\sqrt{s}} e^{-y}$. The function $S_{c}(M, b)$ is the Sudakov form factor, which is universal (process independent) [[]]: it only depends on the type ( $c=q$ or $c=g$ ) of colliding partons, and it resums the logarithmically-enhanced contributions of the form $\ln M^{2} b^{2}$ (the region $q_{T} \ll M$ corresponds to $M b \gg 1$ in impact parameter space). The all-order expression of $S_{c}(M, b)$ is [2]

$$
S_{c}(M, b)=\exp \left\{-\int_{b_{0}^{2} / b^{2}}^{M^{2}} \frac{d q^{2}}{q^{2}}\left[A_{c}\left(\alpha_{\mathrm{S}}\left(q^{2}\right)\right) \ln \frac{M^{2}}{q^{2}}+B_{c}\left(\alpha_{\mathrm{S}}\left(q^{2}\right)\right)\right]\right\},
$$

where $A_{c}\left(\alpha_{\mathrm{S}}\right)$ and $B_{c}\left(\alpha_{\mathrm{S}}\right)$ are perturbative series in $\alpha_{\mathrm{S}}$. The perturbative coefficients $A_{c}^{(1)}, B_{c}^{(1)}, A_{c}^{(2)}$ [[]], $B_{c}^{(2)}$ [Q], 田, [8] and $A_{c}^{(3)}$ [Q] are explicitly known.

The Born level factor ${ }^{2}\left[d \sigma_{c \bar{c}, F}^{(0)}\right]$ in Eq. ([2.3]) is obviously process dependent, although its process dependence is elementary (it is simply due to the Born level scattering amplitude of the partonic process $c \bar{c} \rightarrow F$ ). The remaining process dependence of Eq. ([2.3) is embodied in the 'hard-collinear' factor $\left[H^{F} C_{1} C_{2}\right]$. This factor includes a process-independent part and a processdependent part. The structure of the process-dependent part is the main subject of the present proceeding.

In the case of processes that are initiated at the Born level by the $q \bar{q}$ annihilation channel $(c=q)$, the symbolic factor $\left[H^{F} C_{1} C_{2}\right]$ in Eq. ([2.3) has the following explicit form [ [ل]

$$
\left[H^{F} C_{1} C_{2}\right]_{q \bar{q} ; a_{1} a_{2}}=H_{q}^{F}\left(x_{1} p_{1}, x_{2} p_{2} ; \Omega ; \alpha_{\mathrm{S}}\left(M^{2}\right)\right) C_{q a_{1}}\left(z_{1} ; \alpha_{\mathrm{S}}\left(b_{0}^{2} / b^{2}\right)\right) C_{\bar{q} a_{2}}\left(z_{2} ; \alpha_{\mathrm{S}}\left(b_{0}^{2} / b^{2}\right)\right)
$$

and the functions $H_{q}^{F}$ and $C_{q a}=C_{\bar{q} \bar{a}}$ have the perturbative expansion

$$
\begin{aligned}
H_{q}^{F}\left(x_{1} p_{1}, x_{2} p_{2} ; \Omega ; \alpha_{\mathrm{S}}\right) & =1+\sum_{n=1}^{\infty}\left(\frac{\alpha_{\mathrm{S}}}{\pi}\right)^{n} H_{q}^{F(n)}\left(x_{1} p_{1}, x_{2} p_{2} ; \Omega\right) \\
C_{q a}\left(z ; \alpha_{\mathrm{S}}\right) & =\delta_{q a} \delta(1-z)+\sum_{n=1}^{\infty}\left(\frac{\alpha_{\mathrm{S}}}{\pi}\right)^{n} C_{q a}^{(n)}(z)
\end{aligned}
$$

The function $H_{q}^{F}$ is process dependent, whereas the functions $C_{q a}$ are universal (they only depend on the parton indices). The factorized structure in the right-hand side of Eq. (2.5I) is based on the

\footnotetext{
${ }^{2}$ The cross section at its corresponding lowest order in $\alpha_{\mathrm{S}}$.
} 
following fact: the scale of $\alpha_{\mathrm{S}}$ is $M^{2}$ in the case of $H_{q}^{F}$, whereas the scale is $b_{0}^{2} / b^{2}$ in the case of $C_{q a}$. The appearance of these two different scales is essential [ [5] to disentangle the process dependence of $H_{q}^{F}$ from the process-independent Sudakov form factor $\left(S_{q}\right)$ and collinear functions $\left(C_{q a}\right)$. In the case of processes that start at Born level by the gluon fusion channel $(c=g)$, the physics of the small $-q_{T}$ cross section has a richer structure, which is the consequence of collinear correlations [ 6 ] that are produced by the evolution of the colliding hadrons into gluon partonic states (the interested reader is referred to [6, [2]]). Despite its richer structure, it is possible to disentangle [四] the process dependence of $H_{g}^{F}$ from the process-independent Sudakov form factor $\left(S_{c}\right)$ and collinear tensor functions $\left(C_{g a}^{\mu v}\right)$ analogously to the case of the $q \bar{q}$ channel.

As a consequence of the renormalization-group symmetry (Eqs.(22)-(25), in Ref. [ए2]), the resummation factors $H^{F}, S_{c}$ and $C_{q a}$ are not separately defined (and, thus, computable) in an unambiguous way. Equivalently, each of these separate factors can be precisely defined only by specifying a resummation scheme [[]]. We choose the hard scheme, that is defined as follows. The flavour off-diagonal coefficients $C_{a b}^{(n)}(z)$, with $a \neq b$, are 'regular' functions of $z$ as $z \rightarrow 1$. The $z$ dependence of the flavour diagonal coefficients $C_{q q}^{(n)}(z)$ and $C_{g g}^{(n)}(z)$ in Eqs. (2.7) is instead due to both 'regular' functions and 'singular' distributions in the limit $z \rightarrow 1$. The 'singular' distributions are $\delta(1-z)$ and the customary plus-distributions of the form $\left[\left(\ln ^{k}(1-z)\right) /(1-z)\right]_{+}(k=0,1,2 \ldots)$. The hard scheme is the scheme in which, order-by-order in perturbation theory, the coefficients $C_{a b}^{(n)}(z)$ with $n \geq 1$ do not contain any $\delta(1-z)$ term. We highlight (see also Sect. [3) that this definition directly implies that all the process-dependent virtual corrections to the Born level subprocesses are embodied in the resummation coefficient $H_{c}^{F}$.

We note that the specification of the hard scheme (or any other scheme) has sole practical purposes of presentation (theoretical results can be equivalently presented, as actually done in Refs. [ए]] and [प]], by explicitly parametrizing the resummation-scheme dependence of the resummation factors). The $q_{T}$ cross section, its all-order resummation formula (2.3.3) and any consistent perturbative truncation (either order-by-order in $\alpha_{\mathrm{S}}$ or in classes of logarithmic terms) of the latter [1], [4]] are completely independent of the resummation scheme.

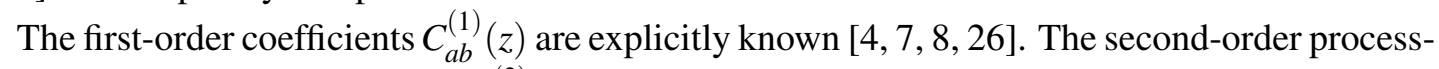

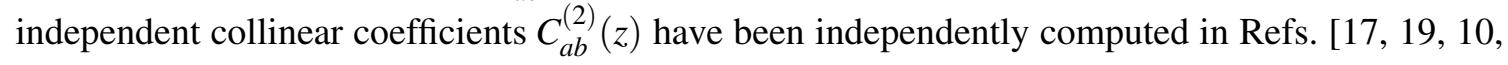
[1] and in Ref. [25] by using two completely different methods, and the results of the two computations are in agreement.

The universality structure of the process-dependent coefficients $H_{c}^{F}$ at NNLO and higher orders (see Sect. [3) is one of the main results that we are discussing in the present proceeding.

\section{Hard-virtual coefficients}

In the hard scheme that we are using, the hard-virtual coefficient contains all the information on the process-dependent virtual corrections, and, therefore, we can show that $H^{F}$ can be related in a process-independent (universal) way to the multiloop virtual amplitude $\mathscr{M}_{c \bar{c} \rightarrow F}$ of the partonic process $c \bar{c} \rightarrow F$.

We consider the partonic elastic-production process

$$
c\left(\hat{p}_{1}\right)+\bar{c}\left(\hat{p}_{2}\right) \rightarrow F\left(\left\{q_{i}\right\}\right),
$$


where the two colliding partons with momenta $\hat{p}_{1}$ and $\hat{p}_{2}$ are either $c \bar{c}=g g$ or $c \bar{c}=q \bar{q}$ and $F\left(\left\{q_{i}\right\}\right)$ is the triggered final-state system in Eq. (메). The loop scattering amplitude of the process in Eq. (B. لل contains ultraviolet (UV) and infrared (IR) singularities, which are regularized in $d=4-2 \varepsilon$ space-time dimensions by using the customary scheme of conventional dimensional regularization. The renormalized all-loop amplitude of the generic process in Eq. (B. 1 ) is denoted by $\mathscr{M}_{c \bar{c} \rightarrow F}$ and it has the perturbative (loop) expansion

$$
\begin{aligned}
& \mathscr{M}_{c \bar{c} \rightarrow F}\left(\hat{p}_{1}, \hat{p}_{2} ;\left\{q_{i}\right\}\right)=\left(\alpha_{\mathrm{S}}\left(\mu_{R}^{2}\right) \mu_{R}^{2 \varepsilon}\right)^{k}\left\{\mathscr{M}_{c \bar{c} \rightarrow F}^{(0)}\left(\hat{p}_{1}, \hat{p}_{2} ;\left\{q_{i}\right\}\right)\right. \\
& \left.+\left(\frac{\alpha_{\mathrm{S}}\left(\mu_{R}^{2}\right)}{2 \pi}\right) \mathscr{M}_{c \bar{c} \rightarrow F}^{(1)}\left(\hat{p}_{1}, \hat{p}_{2} ;\left\{q_{i}\right\} ; \mu_{R}\right)+\sum_{n=3}^{\infty}\left(\frac{\alpha_{\mathrm{S}}\left(\mu_{R}^{2}\right)}{2 \pi}\right)^{n} \mathscr{M}_{c \bar{c} \rightarrow F}^{(n)}\left(\hat{p}_{1}, \hat{p}_{2} ;\left\{q_{i}\right\} ; \mu_{R}\right)\right\},
\end{aligned}
$$

where the value $k$ of the overall power of $\alpha_{\mathrm{S}}$ depends on the specific process. The perturbative terms $\mathscr{M}_{c \bar{c} \rightarrow F}^{(l)}(l=1,2, \ldots)$ are UV finite, but they still depend on $\varepsilon$ (although this dependence is not explicitly denoted in Eq. (B.2)) and, in particular, they are IR divergent as $\varepsilon \rightarrow 0$. The IR divergent contributions to the scattering amplitude have a universal structure [ए]3], which is

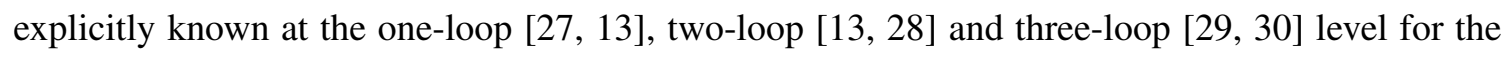
class of processes in Eq. (B.]).

In Ref. [ $[\mathbb{8}]$ we can find the universal (process-independent) relation between the NLO hardvirtual coefficient $H^{F(1)}$ and the leading-order (LO) amplitude $\mathscr{M}_{c \bar{c} \rightarrow F}^{(0)}$ and to the IR finite part of the NLO amplitude $\mathscr{M}_{c \bar{c} \rightarrow F}^{(1)}$. The relation between $H_{c}^{F}$ and $\mathscr{M}_{c \bar{c} \rightarrow F}$ can be extended to NNLO and to higher-order levels [[2]. This extension can be formulated and expressed in simple and general terms by introducing an auxiliary (hard-virtual) amplitude $\widetilde{\mathscr{M}}_{c \bar{c} \rightarrow F}$ that is directly obtained from $\mathscr{M}_{c \bar{c} \rightarrow F}$ in a universal (process-independent) way ${ }^{3}$. In practice, $\widetilde{\mathscr{M}}_{c \bar{c} \rightarrow F}$ is obtained from $\mathscr{M}_{c \bar{c} \rightarrow F}$ by removing its IR divergences and a definite amount of IR finite terms. The (IR divergent and finite) terms that are removed from $\mathscr{M}_{c \bar{c} \rightarrow F}$ originate from real emission contributions to the cross section and, therefore, these terms and $\widetilde{\mathscr{M}}_{c \bar{c} \rightarrow F}$ specifically depend on the transverse-momentum cross section of Eq. (2.]). The relation between $H_{c}^{F}$ and $\mathscr{M}_{c \bar{c} \rightarrow F}$ is based on an universal all-order factorization formula [ㄹ] that emerges from the factorization properties of soft (and collinear) parton radiation. We have explicitly determined this relation up to the NNLO [12]. More precisely, we have shown [D2] that this relation is fully determined by the structure of IR singularities of the all-order amplitude $\mathscr{M}_{\bar{c} \rightarrow F}$ and by renormalization-group invariance up to a single coefficient (of soft origin) at each perturbative order.

We can relate the subtracted amplitude $\widetilde{\mathscr{M}}_{c \bar{c} \rightarrow F}$ to the process-dependent resummation coefficients $H_{c}^{F}$ of Eqs. ([2.3]) and ([2.5)). For processes initiated by $q \bar{q}$ annihilation (see Eqs. ([2.5]) and (L.6)), the all-order coefficient $H_{q}^{F}$ can be written as

$$
\alpha_{\mathrm{S}}^{2 k}\left(M^{2}\right) H_{q}^{F}\left(x_{1} p_{1}, x_{2} p_{2} ; \Omega ; \alpha_{\mathrm{S}}\left(M^{2}\right)\right)=\frac{\left|\widetilde{\mathscr{M}}_{q \bar{q} \rightarrow F}\left(x_{1} p_{1}, x_{2} p_{2} ;\left\{q_{i}\right\}\right)\right|^{2}}{\left|\mathscr{M}_{q \bar{q} \rightarrow F}^{(0)}\left(x_{1} p_{1}, x_{2} p_{2} ;\left\{q_{i}\right\}\right)\right|^{2}},
$$

where $k$ is the value of the overall power of $\alpha_{\mathrm{S}}$ in the expansion of $\mathscr{M}_{\bar{c} \rightarrow F}$ (see Eq. (B.2)).

The expression (B.3) allows us the explicit computation of the process-dependent resummation coefficients $H_{c}^{F}$ for an arbitrary process of the class in Eq. (ㅍ. $\mathbb{d}$ ). The computation of $H_{c}^{F}$ up to the

\footnotetext{
${ }^{3}$ The interested reader is referred to [미], where there are all the formulae to obtain $\widetilde{\mathscr{M}}_{c \bar{c} \rightarrow F}$.
} 
NNLO is straightforward, provided the scattering amplitude $\mathscr{M}_{\bar{c} \rightarrow F}$ of the corresponding partonic subprocess is available (known) up to the NNLO (two-loop) level.

Some examples (DY and Higgs boson production) are explicitly reported in Appendix A of Ref. [12]. In particular, in Appendix A of Ref. [12], we used Eq. (3.3]), and we presented the explicit expression of the NNLO hard-virtual coefficient $H_{q}^{\gamma \gamma(2)}$ for the process of diphoton production []] . Recently, Eq. (3.3]) was used to obtain the hard-virtual factor in the case of Higgs production in bottom quark annihilation [B] $]$, in order to calculate the transverse momentum distribution at NNLO+NNLL.

The same procedure that was applied to derive the universal formula for the hard-virtual coefficient $H_{c}^{F}$ can be used within the related formalism of threshold resummation [B2] for the total cross section. The process-independent formalism of threshold resummation also involves a corresponding process-dependent hard factor which has a universality structure [ए2] that is analogous to the case of transverse-momentum resummation. Recently, we also extended the threshold resummation results of Ref. [12] to the next subsequent order $\left(\mathrm{N}^{3} \mathrm{LL}\right)$ [33]. The general (process-independent) $\mathrm{N}^{3} \mathrm{LL}$ results of Ref. [33] are based on the universality structure of the hard-virtual factor, and they exploit the recent computation of the $\mathrm{N}^{3}$ LO Higgs boson cross section [34] within the soft-virtual approximation. For the specific case of DY production we confirm [33] the soft-virtual $\mathrm{N}^{3} \mathrm{LO}$ results of Ref. [BS]].

The results enumerated in this proceeding, with the knowledge of the other process-independent resummation coefficients, complete the $q_{T}$ resummation formalism in explicit form up to full NNLL and NNLO accuracy for all the processes in the class of Eq. (ل्. $)$. This allows applications to NNLL+NNLO resummed calculations for any processes whose NNLO scattering amplitudes are available. Moreover, we have all the ingredients to implement the $q_{T}$ subtraction formalism [1]7] straightforwardly, to perform fully-exclusive NNLO computations for each of these processes.

\section{References}

[1] Y. L. Dokshitzer, D. Diakonov and S. I. Troian, Phys. Lett. B 79 (1978) 269, Phys. Rep. A 58 (1980) 269.; G. Parisi and R. Petronzio, Nucl. Phys. B 154 (1979) 427.; G. Curci, M. Greco and Y. Srivastava, Nucl. Phys. B 159 (1979) 451.; J. C. Collins and D. E. Soper, Nucl. Phys. B 193 (1981) 381 [Erratum-ibid. B 213 (1983) 545], Nucl. Phys. B 197 (1982) 446.

[2] J. C. Collins, D. E. Soper and G. Sterman, Nucl. Phys. B 250 (1985) 199.

[3] J. Kodaira and L. Trentadue, Phys. Lett. B 112 (1982) 66, report SLAC-PUB-2934 (1982), Phys. Lett. B 123 (1983) 335.; S. Catani, E. D'Emilio and L. Trentadue, Phys. Lett. B 211 (1988) 335.

[4] D. de Florian and M. Grazzini, Phys. Rev. Lett. 85 (2000) 4678 [arXiv:hep-ph/0008152].

[5] S. Catani, D. de Florian and M. Grazzini, Nucl. Phys. B 596 (2001) 299 [arXiv:hep-ph/0008184].

[6] S. Catani and M. Grazzini, Nucl. Phys. B 845 (2011) 297 [arXiv:1011.3918 [hep-ph]].

[7] C. T. H. Davies and W. J. Stirling, Nucl. Phys. B 244 (1984) 337; C. T. H. Davies, B. R. Webber and W. J. Stirling, Nucl. Phys. B 256 (1985) 413.

[8] D. de Florian and M. Grazzini, Nucl. Phys. B 616 (2001) 247 [arXiv:hep-ph/0108273].

[9] T. Becher, M. Neubert, Eur. Phys. J. C71 (2011) 1665 [arXiv:1007.4005 [hep-ph]]; T. Becher, M. Neubert and D. Wilhelm, JHEP 1305 (2013) 110 [arXiv:1212.2621 [hep-ph]]. 
[10] S. Catani and M. Grazzini, Eur. Phys. J. C 72 (2012) 2013 [Erratum-ibid. C 72 (2012) 2132] [arXiv:1106.4652 [hep-ph]].

[11] S. Catani, L. Cieri, D. de Florian, G. Ferrera and M. Grazzini, Eur. Phys. J. C 72 (2012) 2195 [arXiv:1209.0158 [hep-ph]].

[12] S. Catani, L. Cieri, D. de Florian, G. Ferrera and M. Grazzini, Nucl. Phys. B 881 (2014) 414 [arXiv:1311.1654 [hep-ph]].

[13] S. Catani, Phys. Lett. B 427 (1998) 161 [hep-ph/9802439].

[14] G. Bozzi, S. Catani, D. de Florian and M. Grazzini, Nucl. Phys. B 737 (2006) 73 [arXiv:hep-ph/0508068].; D. de Florian, G. Ferrera, M. Grazzini and D. Tommasini, JHEP 1111 (2011) 064 [arXiv:1109.2109 [hep-ph]], JHEP 1206 (2012) 132 [arXiv:1203.6321 [hep-ph]]; J. Wang, C. S. Li, Z. Li, C. P. Yuan and H. T. Li, Phys. Rev. D 86 (2012) 094026 [arXiv:1205.4311 [hep-ph]].

[15] G. Bozzi, S. Catani, G. Ferrera, D. de Florian and M. Grazzini, Phys. Lett. B 696 (2011) 207 [arXiv:1007.2351 [hep-ph]].

[16] M. Guzzi, P. M. Nadolsky and B. Wang, arXiv:1309.1393 [hep-ph].

[17] S. Catani and M. Grazzini, Phys. Rev. Lett. 98 (2007) 222002 [arXiv:hep-ph/0703012].

[18] M. Grazzini, JHEP 0802 (2008) 043 [arXiv:0801.3232 [hep-ph]].

[19] S. Catani, L. Cieri, G. Ferrera, D. de Florian and M. Grazzini, Phys. Rev. Lett. 103 (2009) 082001 [arXiv:0903.2120 [hep-ph]].

[20] G. Ferrera, M. Grazzini and F. Tramontano, Phys. Rev. Lett. 107 (2011) 152003 [arXiv:1107.1164 [hep-ph]].

[21] S. Catani, L. Cieri, D. de Florian, G. Ferrera and M. Grazzini, Phys. Rev. Lett. 108 (2012) 072001 [arXiv:1110.2375 [hep-ph]].

[22] M. Grazzini, S. Kallweit, D. Rathlev and A. Torre, report ZU-TH-21-13 (arXiv:1309.7000 [hep-ph]).

[23] F. Cascioli, T. Gehrmann, M. Grazzini, S. Kallweit, P. MaierhÃúfer, A. von Manteuffel, S. Pozzorini and D. Rathlev et al., arXiv:1405.2219 [hep-ph].

[24] M. G. Echevarria, A. Idilbi and I. Scimemi, JHEP 1207 (2012) 002 [arXiv:1111.4996 [hep-ph]], Phys. Lett. B 726 (2013) 795 [arXiv:1211.1947 [hep-ph]]; J. C. Collins and T. C. Rogers, Phys. Rev. D 87 (2013) 3, 034018 [arXiv:1210.2100 [hep-ph]].

[25] T. Gehrmann, T. Lubbert and L. L. Yang, Phys. Rev. Lett. 109 (2012) 242003 [arXiv:1209.0682 [hep-ph]], arXiv:1403.6451 [hep-ph].

[26] R. P. Kauffman, Phys. Rev. D 45 (1992) 1512; C. P. Yuan, Phys. Lett. B 283 (1992) 395.

[27] W. T. Giele and E. W. N. Glover, Phys. Rev. D 46 (1992) 1980; Z. Kunszt, A. Signer and Z. Trocsanyi, Nucl. Phys. B 420 (1994) 550 [hep-ph/9401294]; S. Catani and M. H. Seymour, Nucl. Phys. B 485 (1997) 291 [Erratum-ibid. B 510 (1998) 503] [hep-ph/9605323].

[28] R. V. Harlander, Phys. Lett. B 492 (2000) 74 [hep-ph/0007289]; V. Ravindran, J. Smith and W. L. van Neerven, Nucl. Phys. B 704 (2005) 332 [hep-ph/0408315]. 
[29] S. Moch, J. A. M. Vermaseren and A. Vogt, JHEP 0508 (2005) 049 [hep-ph/0507039], Phys. Lett. B 625 (2005) 245 [hep-ph/0508055]; P. A. Baikov, K. G. Chetyrkin, A. V. Smirnov, V. A. Smirnov and M. Steinhauser, Phys. Rev. Lett. 102 (2009) 212002 [arXiv:0902.3519 [hep-ph]]; R. N. Lee,

A. V. Smirnov and V. A. Smirnov, JHEP 1004 (2010) 020 [arXiv:1001.2887 [hep-ph]]; T. Gehrmann, E. W. N. Glover, T. Huber, N. Ikizlerli and C. Studerus, JHEP 1006 (2010) 094 [arXiv:1004.3653 [hep-ph]].

[30] L. J. Dixon, L. Magnea and G. F. Sterman, JHEP 0808 (2008) 022 [arXiv:0805.3515 [hep-ph]]; T. Becher and M. Neubert, JHEP 0906 (2009) 081 [arXiv:0903.1126 [hep-ph]].

[31] R. V. Harlander, A. Tripathi and M. Wiesemann, arXiv:1403.7196 [hep-ph]; A. Tripathi, these proceedings.

[32] G. F. Sterman, Nucl. Phys. B 281 (1987) 310; S. Catani and L. Trentadue, Nucl. Phys. B 327 (1989) 323, Nucl. Phys. B 353 (1991) 183.

[33] S. Catani, L. Cieri, D. de Florian, G. Ferrera and M. Grazzini, arXiv:1405.4827 [hep-ph].

[34] C. Anastasiou, C. Duhr, F. Dulat, E. Furlan, T. Gehrmann, F. Herzog and B. Mistlberger, arXiv:1403.4616 [hep-ph].

[35] T. Ahmed, M. Mahakhud, N. Rana and V. Ravindran, arXiv:1404.0366 [hep-ph]. 